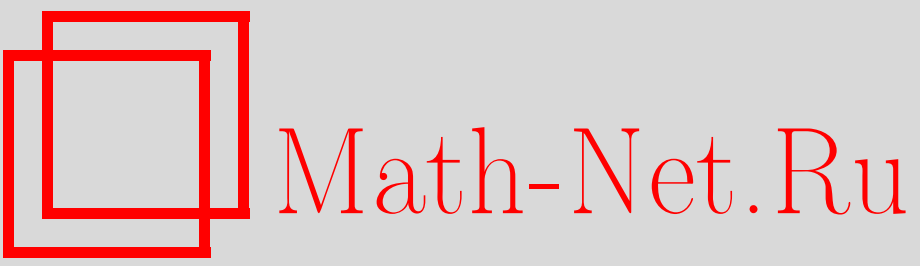

Г. В. Ефимов, Амплитуды при высоких энергиях в нелокальной теории, ТМФ, 2001, том 128, номер 3, 395-402

DOI: https://doi.org/10.4213/tmf503

Использование Общероссийского математического портала Math-Net.Ru подразумевает, что вы прочитали и согласны с пользовательским соглашением

http://www.mathnet.ru/rus/agreement

Параметры загрузки:

IP : 54.147 .182 .235

26 апреля 2023 г., 16:08:57 
ТЕОРЕТИЧЕСКАЯ

И МАТЕМАТИЧЕСКАЯ

ФИЗИКА

Том 128, № 3

сентябрь, 2001

(C) 2001 г.

Г.В. Ефимов ${ }^{*}$

\section{АМПЛИТУДЫ ПРИ ВЫСОКИХ ЭНЕРГИЯХ В НЕЛОКАЛЬНОЙ ТЕОРИИ}

В нелокальной квантовой теории скалярного поля, допускающей рост амплитуд $\sim e^{B\left\|p^{2}\right\|^{\rho}}$ в комплексной $p^{2}$-плоскости, получено ограничение на полное поперечное сечение рассеяния: $\sigma_{\text {tot }}(s) \leqslant C s^{\rho-1} \ln ^{2} s$.

\section{1. ВВЕДЕНИЕ}

Поведение амплитуд рассеяния при высоких энергиях является одной из центральных проблем физики элементарных частиц. Стандартные методы квантовой теории поля надежны при вычислениях по теории возмушений, лишь когда энергии взаимодействующих частиц не столь высоки и эффективные константы связи малы. При увеличении энергии эффективные константы связи растут, так что следующие поправки по теории возмушений начинают играть определяюшую роль. В настояшее время используются различные приближения, связанные с суммированием определенных типов диаграмм Фейнмана при помоши метода ренормгруппы. Однако методы суммирования рядов теории возмушений еше далеки от завершенности. Поэтому крайне важно знать строгие ограничения на поведение амплитуд рассеяния при высоких энергиях, следуюшие из так называемых первых принципов, т.е. из обших аксиоматических основ квантовой теории поля.

Стандартный путь доказательства хорошо известного ограничения Фруассара на полное поперечное сечение в локальной квантовой теории скалярного поля основан на комбинации трех условий: унитарности, аналитичности амплитуды упругого рассеяния в эллипсе Мартена-Лемана и полиномиальной ограниченности амплитуд в локальной квантовой теории поля (см., например, [1], [2]). Поэтому ограничение Фруассара рассматривается как внутреннее свойство локальной квантовой теории поля.

В данной статье получено ограничение на амплитуду упругого рассеяния при высоких энергиях в нелокальной квантовой теории поля (НКТП), в которой рост амплитудв комплексной области импульсных переменных больше линейной экспоненты по импульсу: так называемые сушественно нелокализуемые полевые теории [3], [4]

$$
\mathcal{A} \sim e^{\left(l^{2} p^{2}\right)^{\rho}}, \quad \rho \geqslant 1 .
$$

\footnotetext{
* Объединенный институт ядерных исследований, Дубна, Московская обл., Россия
} 
Параметр $l$ определяет масштаб области нелокального взаимодействия.

Исторически НКТП развивалась как физически приемлемая попытка устранить ультрафиолетовые расходимости, связанные с локальным характером взаимодействия, и не имевшая непосредственного приложения к физике элементарных частиц. В настоящее время, однако, в рамках методов НКТП удается описать кварки и глюоны в области конфайнмента. Так предположение о том, что пропагаторы кварков описьваются целыми функциями первого порядка роста в комплексной $p^{2}$-плоскости, лежит в основе виртонной модели и модели конфайнмированных кварков [5]. Предположение, что вакуум КХД реализуется самодуальным однородным вакуумным глюонным полем, приводит к аналитическому конфайнменту кварков и глюонов [6]. Другими словами, то, что пропагаторы кварков и глюонов являются целыми аналитическими функциями в комплексной плоскости $p^{2}$, гарантирует конфайнмент, т.е. кварки и глюоны не могут существовать в свободном состоянии как обычные частищы, однако сушествуют как переносяшие взаимодействие виртуальные частицы. При этом $S$-матрица унитарна на пространстве физических состояний - адронов. Расчеты показали, что перечисленные модели правильно описывают физику адронов при низких энергиях [7]. Следует также отметить, что аналитический конфайнмент в упомянутых моделях реализуется именно целыми функциями первого порядка роста ( $\rho=1$ в формуле $(1))$, так что есть основания считать, что случай с $\rho=1$ играет выделенную роль в физике частиц.

Однако существует сильное предубеждение против релятивистской НКТП. Главное возражение состоит в том, что формфакторы (см. [5], [8]), пропагаторы и амплитуды (см. [6]), которые являются целыми аналитическими функциями, убывающими в евклидовом импульсном пространстве, быстро растут в физической области. Коэффициенты разложения по теории возмушений для любой амплитуды растут в физической области каK

$$
\mathcal{M}\left(s_{i j}\right)=\sum_{n=0}^{\infty} g^{n_{0}+2 n} \mathcal{M}^{(n)}\left(s_{i j}\right), \quad \mathcal{M}^{(n)}\left(s_{i j}\right) \sim e^{n s_{i j}^{\rho}} \quad \text { при } \quad s_{i j} \rightarrow+\infty
$$

где $s_{i j}=\left(k_{i}+k_{j}\right)^{2}$ и $\rho \geqslant 1$.

Это означает, что режим сильной константы связи в нелокальной теории наступает при $g^{2} e^{\left(l^{2} s\right)^{\rho}} \geqslant 1$, т.е. при относительно невысоких энергиях, сравнимых с величиной области конфайнмента $s$, приблизительно равной 1 ГэВ ${ }^{2}$. Следовательно, нельзя пользоваться вычислениями по теории возмущений при более высоких энергиях. Необходимо привлекать методы суммирования рядов теории возмушений, что в настояшее время представляется весьма сложной задачей. В локальных перенормируемых теориях режим сильной связи наступает при $g^{2} \ln s \geqslant 1$ и с практической точки зрения эта гранища весьма далека.

Однако следует напомнить и подчеркнуть, что математическое доказательство существования НКТП находится на том же уровне строгости, что и локальной КТП. Аксиоматическая теория НКТП сформулирована в [3], [4], где доказаны основные теоремы аксиоматического подхода. Лагранжев формализм развит в [8], где в каждом порядке теории возмущений была доказана унитарность $S$-матрицы и было показано, что аналитические свойства амплитуд в любой конечной области комплексных импульсных пе- 
ременных в локальной и нелокальной теориях совпадают.

Для того чтобы получить ограничение сверху на полное поперечное сечение в НКТП, необходимо предположить, что сушествует такой метод суммирования рядов теории возмушений, что точная $S$-матрица унитарна и аналитические особенности амплитуд в любой конечной области комплексных импульсных переменных в локальной и нелокальной теориях совпадают. В частности, амплитуда упругого рассеяния аналитична в эллипсе Мартена-Лемана. Кстати, то же самое предположение делается и в локальной КТП. Первый результат для класса существенно нелокализуемых теорий, в которых рост амплитуд во всем импульсном пространстве ограничен экспонентой $e^{b\left\|p^{2}\right\|^{\rho}}$, $\rho>1 / 2$, был получен в [9]. Авторы показали, что полное поперечное сечение ограничено функцией

$$
\sigma_{\text {tot }}(s)<C s^{1+2 \rho} \text {. }
$$

Эта оценка представляется весьма завьшенной. С физической точки зрения при высоких энергиях полное поперечное сечение при нелокальном взаимодействии, по крайней мере, не должно расти быстрее поперечного сечения при локальном взаимодействии, поскольку "нелокальные частицы", имеющие некоторую протяженность, должны становиться все более и более прозрачными.

В этой работе показано, что ограничение Фруассара на полное поперечное сечение в нелокальной теории при $\rho=1$ то же самое, что и в локальной теории. Наша основная идея состоит в следуюшем. При доказательстве в работе [9] используется ограниченность амплитуд в нелокальной теории во всей области комплексной переменной, в то время как нам достаточно знать ограничение на амплитуду упругого рассеяния только при вещественных и положительных $s$ и в малой комплексной окрестности $t=0$. Для получения этого ограничения $S$-матрицу следует рассматривать как унитарный оператор на пространстве Фока физических состояний.

\section{2. УНИТАРНОСТЬ НА МАССОВОЙ ПОВЕРХНОСТИ}

Для простоты будем рассматривать однокомпонентное скалярное поле. Обобшение на другие поля не вносит никаких принципиально новых трудностей.

Пространство Фока $\mathcal{F}$ физических частищ состоит из векторов

$$
F=f_{0}|0\rangle+\sum_{n=1}^{\infty} \int \prod_{j=1}^{n} d \vec{k}_{j} f_{n}(\vec{k})\left|\vec{k}_{1}, \ldots, \vec{k}_{n}\right\rangle
$$

где

$$
\left|\vec{k}_{1}, \ldots, \vec{k}_{n}\right\rangle=\frac{1}{\sqrt{n !}} a_{\vec{k}_{1}}^{+} \cdot \ldots \cdot a_{\vec{k}_{n}}^{+}|0\rangle
$$

Вектор $|0\rangle$ описывает вакуум, нормированный условием $\langle 0 \mid 0\rangle=1$. Норма состояния $F \in \mathcal{F}$ определяется как

$$
\|F\|^{2}=\langle F, F\rangle=\left|f_{0}\right|^{2}+\sum_{n=1}^{\infty} \int \prod_{j=1}^{n} d \vec{k}_{j}\left|f_{n}(\vec{k})\right|^{2}<\infty
$$


$S$-матрица может быть представлена в форме

$$
\begin{aligned}
S=I+i T \\
T=\sum_{n_{1}=2}^{\infty} \sum_{n_{2}=2}^{\infty} \int \prod_{j=1}^{n_{1}} \frac{d \vec{k}_{j}}{\sqrt{2 \omega_{j}}} \int \prod_{l=1}^{n_{2}} \frac{d \vec{p}_{l}}{\sqrt{2 \omega_{l}}} \times \\
\quad \times \frac{1}{\sqrt{n_{1} !}} a_{\vec{k}_{1}}^{+} \ldots \cdot a_{\vec{k}_{n_{1}}}^{+} T_{n_{1} n_{2}}(k, p) a_{\vec{p}_{1}} \cdot \ldots \cdot a_{\vec{p}_{n_{2}}} \frac{1}{\sqrt{n_{2} !}}
\end{aligned}
$$

где

$$
\begin{gathered}
k_{j}=\left(\omega_{j}, \vec{k}_{j}\right), \quad \omega_{j}=\sqrt{m^{2}+\vec{k}_{j}^{2}}, \quad \vec{k}_{j} \in \mathbf{R}^{3}, \\
T_{n_{1} n_{2}}(k, p)=\sum_{v=0}^{\min \left(n_{1}, n_{2}\right)} \sum_{\{a, b\}} M_{a_{1} b_{1}}(k, p) \cdot \ldots \cdot M_{a_{v} b_{v}}(k, p), \\
M_{a b}(k, p)=\delta^{(4)}\left(\sum_{j=1}^{a} k_{j}-\sum_{l=1}^{b} p_{l}\right) \mathcal{M}_{a b}(k, p) .
\end{gathered}
$$

Предполагается, что $S$-матрица перенормирована и удовлетворяет условию стабильности:

$$
S|0\rangle=|0\rangle, \quad S|\vec{k}\rangle=|\vec{k}\rangle
$$

т.е. вакуумные петли устранены и одночастичные состояния перенормированы. Функции $\mathcal{M}_{a b}(k, p)$ состоят из связанных диаграмм Фейнмана и зависят от релятивистски-инвариантных импульсных переменных на массовой поверхности:

$$
s_{i j}=\left(k_{i}-k_{j}\right)^{2}>0, \quad s_{l m}=\left(p_{l}-p_{m}\right)^{2}>0, \quad t_{i l}=\left(k_{i}-p_{l}\right)^{2}<0,
$$

где $i, j=1, \ldots, b$ и $l, m=1, \ldots, a$.

Если $S$-матрица унитарна:

$$
S^{+} S=S S^{+}=I
$$

то мы имеем очевидное неравенство

$$
\|T\|=\|S-I\| \leqslant\|S\|+\|I\| \leqslant 2,
$$

откуда следует, что неравенство

$$
|\langle F, T F\rangle| \leqslant 2\langle F, F\rangle
$$

справедливо для любого $F \in \mathcal{F}$. Это неравенство является главным инструментом нашего исследования. 


\section{3. УПРУГОЕ РАССЕЯНИЕ}

Рассмотрим амплитуду упругого рассеяния. Введем вектор

$$
F=\iint d \vec{k}_{1} d \vec{k}_{2} f\left(\vec{k}_{1}, \vec{k}_{2}\right)\left|\vec{k}_{1}, \vec{k}_{2}\right\rangle
$$

с нормой

$$
\langle F, F\rangle=\iint d \vec{k}_{1} d \vec{k}_{2}\left|f\left(\vec{k}_{1}, \vec{k}_{2}\right)\right|^{2} .
$$

Тогда подмодульное выражение в левой части неравенства (4) имеет вид

$$
\begin{aligned}
\langle F, T F\rangle= & \iint \frac{d \vec{k}_{1} d \vec{k}_{2}}{\sqrt{2 \omega_{1} 2 \omega_{2}}} \iint \frac{d \vec{p}_{1} d \vec{p}_{2}}{\sqrt{2 E_{1} 2 E_{2}}} \delta^{(4)}\left(k_{1}+k_{2}-p_{1}-p_{2}\right) \times \\
& \times f\left(\vec{k}_{1}, \vec{k}_{2}\right) \mathcal{M}(s, t) f\left(\vec{p}_{1}, \vec{p}_{2}\right),
\end{aligned}
$$

где $\mathcal{M}(s, t)=\mathcal{M}_{22}\left(k_{1}, k_{2} ; p_{1}, p_{2}\right), s=\left(k_{1}+k_{2}\right)^{2}$ и $t=\left(k_{1}-p_{1}\right)^{2}$.

Чтобы выделить поведение амплитуды $\mathcal{M}(s, t)$ при $s \rightarrow \infty$, введем функцию

$$
f\left(\vec{k}_{1}, \vec{k}_{2}\right)=\frac{1}{\kappa^{3}} \phi\left(\frac{\left(\vec{k}_{1}-\vec{q}\right)^{2}}{\kappa^{2}}\right) \phi\left(\frac{\left(\vec{k}_{2}+\vec{q}\right)^{2}}{\kappa^{2}}\right),
$$

где $\phi(u)$ - положительная функция с ограниченным носителем

$$
\operatorname{supp} \phi(u)=\left\{u: 0<u<\frac{1}{2}\right\}
$$

и нормировкой

$$
\int \frac{d \vec{k}}{\kappa^{3}} \phi^{2}\left(\frac{\vec{k}^{2}}{\kappa^{2}}\right)=2 \pi \int_{0}^{\frac{1}{2}} d u \sqrt{u} \phi^{2}(u)=1,
$$

так что $\langle F, F\rangle=1$. Параметры $\vec{q}$ и $\kappa$ выбраны таким образом, что

$$
Q^{2}=\vec{q}^{2}+m^{2} \gg m^{2}, \quad \kappa^{2} \ll m^{2} \ll Q^{2} .
$$

Для $\kappa \rightarrow 0$ имеем

$$
\left|f\left(\vec{k}_{1}, \vec{k}_{2}\right)\right|^{2} \rightarrow \delta\left(\vec{k}_{1}-\vec{q}\right) \delta\left(\vec{k}_{2}+\vec{q}\right) .
$$

Введем новые переменные в интеграле (6), с определенной в (7) функцией $f$ :

$$
\vec{k}_{1} \rightarrow \vec{k}_{1}+\vec{q}, \quad \vec{k}_{2} \rightarrow \vec{k}_{2}-\vec{q}, \quad \vec{p}_{1} \rightarrow \vec{p}_{1}+\vec{q}, \quad \vec{p}_{2} \rightarrow \vec{p}_{2}-\vec{q}
$$

Область интегрирования ограничена условиями

$$
\left(\vec{k}_{j}\right)^{2} \leqslant \frac{\kappa^{2}}{2}, \quad\left(\vec{p}_{j}\right)^{2} \leqslant \frac{\kappa^{2}}{2}, \quad j=1,2
$$


Для больших $Q \gg m^{2}$ имеем

$$
\begin{gathered}
\omega_{ \pm}=\sqrt{m^{2}+(\vec{k} \pm \vec{q})^{2}}=Q \pm(\vec{k} \vec{n})+O\left(\frac{\kappa}{Q^{2}}\right)=Q\left[1+O\left(\frac{\sqrt{\kappa^{2}}}{Q}\right)\right], \\
\vec{n}=\frac{\vec{q}}{Q} \rightarrow \frac{\vec{q}}{|\vec{q}|}, \quad(\vec{n})^{2}=\frac{\vec{q}^{2}}{Q^{2}} \rightarrow 1 .
\end{gathered}
$$

При $Q \rightarrow \infty$ получаем

$$
\begin{aligned}
\langle F, T F\rangle \rightarrow & \frac{1}{\left(\pi \kappa^{2}\right)^{3} s} \iint d \vec{k}_{1} d \vec{k}_{2} \iint d \vec{p}_{1} d \vec{p}_{2} \times \\
& \times \phi\left(\frac{\vec{k}_{1}^{2}}{\kappa^{2}}\right) \phi\left(\frac{\vec{k}_{2}^{2}}{\kappa^{2}}\right) \phi\left(\frac{\vec{p}_{1}^{2}}{\kappa^{2}}\right) \phi\left(\frac{\vec{p}_{2}^{2}}{\kappa^{2}}\right) \times \\
& \times \delta^{(3)}\left(\vec{k}_{1}+\vec{k}_{2}-\vec{p}_{1}-\vec{p}_{2}\right) \delta\left(\left(\vec{k}_{1}-\vec{k}_{2}-\vec{p}_{1}+\vec{p}_{2}\right) \vec{n}\right) \times \\
& \times \mathcal{M}\left(s,-\left[\left(\vec{k}_{1}-\vec{p}_{1}\right)^{2}-\left(\vec{n}\left(\vec{k}_{1}-\vec{p}_{1}\right)\right)^{2}\right]\right)= \\
= & \frac{\pi \kappa^{2}}{2 s} \int_{0}^{1} d u \Phi^{2}(u) \mathcal{M}\left(s,-u \kappa^{2}\right) .
\end{aligned}
$$

Положительная функция $\Phi(u)$ удовлетворяет условиям

$$
\begin{gathered}
\Phi(u)=\left.\int d \vec{l} \phi\left(\vec{l}^{2}\right) \phi\left((\vec{l}+\vec{r})^{2}\right)\right|_{\vec{r}^{2}=u}, \\
\operatorname{supp} \Phi(u)=\{u: 0 \leqslant u \leqslant 1\}, \quad \Phi(0)=1, \quad \Phi(u) \leqslant 1 .
\end{gathered}
$$

Таким образом, при больших $s \rightarrow \infty$ неравенство (4) для положительных функций $\Phi(u)$ и любых значений параметра $\kappa^{2}<s$ выглядит следуюшим образом:

$$
\left|\kappa^{2} \int_{0}^{1} d u \Phi^{2}(u) \mathcal{M}\left(s,-u \kappa^{2}\right)\right| \leqslant \frac{4}{\pi} s, \quad 0<\kappa^{2}<s .
$$

\section{4. НЕЛОКАЛЬНАЯ КВАНТОВАЯ ТЕОРИЯ ПОЛЯ}

Будем предполагать, что $S$-матрица (2) содержит все точные амплитуды. Другими словами, сушествует такой метод суммирования рядов теории возмушений, что:

a) $S$-матрица унитарна;

б) аналитические свойства амплитуд в любой конечной области импульсного комплексного пространства совпадают в локальной и нелокальной квантовых теориях поля;

в) амплитуды $M_{n}\left(x_{1}, \ldots, x_{n}\right)$ в $x$-пространстве являются обобшенными функциями, которые определены на пространстве основных функций $Z_{\gamma}$, (см. [3], [4], [8]), состоящем из целых аналитических функций, которые удовлетворяют неравенству

$$
\left|f\left(x_{1}+i y_{1}, \ldots, x_{n}+i y_{n}\right)\right| \leqslant A \exp \left(\sum_{i=1}^{n}\left[-a_{i}\left|x_{i}\right|^{2 \gamma}+b_{i}\left|y_{i}\right|^{2 \gamma}\right]\right)
$$


где $A, a_{i}$ и $b_{i}$ - положительные постоянные и $\gamma>1$;

г) $\widetilde{Z}_{\gamma}=Z_{\rho}$ при $2 \rho=2 \gamma /(2 \gamma-1)>1$;

д) амплитуды $M_{n}\left(p_{1}, \ldots, p_{n}\right)$ в комплексном импульсном $p^{2}$-пространстве удовлетворяют неравенству

$$
\left|M_{n}\left(p_{1}, \ldots, p_{n}\right)\right| \leqslant A \exp \left(\sum_{i, j=1}^{n} A_{i j}\left|\left(p_{i} p_{j}\right)\right|^{\rho}\right)
$$

где $A$ и $A_{i j}$ - постоянные.

Амплитуда упругого рассеяния $\mathcal{M}(s, t)$ для больших вешественных положительных $s$ и малых вешественных $t$ может быть оценена как

$$
|\mathcal{M}(s, t)| \simeq R(s) e^{h(s) t}
$$

где

$$
R(s) \leqslant A e^{a s^{\rho}}, \quad h(s) \leqslant b s^{\rho-1}
$$

и $A, a$ и $b$-постоянные. Из оценки (11) и неравенства (8) следует, что

$$
\frac{R(s)}{h(s)} \leqslant C s, \quad R(s) \leqslant C s^{\rho} .
$$

Другими словами, для больших вешественных положительных $s$ и малых вешественных $t$ имеем

$$
|\mathcal{M}(s, t)| \leqslant A s^{\rho}, \quad|t| \leqslant \frac{B}{s^{\rho-1}}
$$

\section{5. ОГРАНИЧЕНИЕ ФРУАССАРА}

Вывод ограничения Фруассара на полное поперечное сечение в локальной теории приведен в [1]. Выведем ограничение Фруассара для рассматриваемого нами случая. Мнимая часть амплитуды упругого рассеяния записывается как

$$
\mathcal{I}(s, t)=\frac{1}{8 \pi} \operatorname{Im} \mathcal{M}(s, t)=\frac{s^{\frac{1}{2}}}{k} \sum_{l=0}^{\infty}(2 l+1) a_{l}(s) P_{l}(\cos \theta),
$$

где $\cos \theta=1+2 t /\left(s-4 m^{2}\right), t<0$.

Предполагаются справедливыми следующие утверждения:

а) условие унитарности

$$
0 \leqslant a_{l}(s) \leqslant 1
$$

б) функция $\mathcal{M}(s, t)$ аналитична в эллипсе Мартэна-Лемана, задаваемом условием

$$
|\cos \theta|<1+\frac{8 m^{2}}{s-4 m^{2}}
$$

в) справедливо неравенство (12). 
Воспользуемся неравенством

$$
P_{l}(x)>\frac{C}{\sqrt{1+l}}(1+\sqrt{2 x-2})^{l} \sim \frac{C}{\sqrt{l}}\left(1+\frac{b}{s^{\frac{\rho}{2}}}\right)^{l}
$$

при $x=1+t / s \sim 1+B / s^{\rho}>1$ (здесь и далее знак $\sim$ означает асимптотику при больших $s$ ). При $t_{0}=B / s^{\rho-1}$ имеем соотношение

$$
C \sqrt{l} a_{l}(s)\left(1+\frac{b}{s^{\frac{\rho}{2}}}\right)^{l}<2 l a_{l}(s) P_{l}\left(1+\frac{t_{0}}{s}\right)<s^{\rho},
$$

поэтому

$$
a_{l}(s)<\frac{C}{\sqrt{l}} \exp \left(\rho \ln s-\frac{c l}{s^{\frac{\rho}{2}}}\right) .
$$

Величина $a_{l}(s) \ll 1$ для $l>L=C s^{\rho / 2} \ln s$, откуда следует

$$
\mathcal{I}(s, 0)<\frac{s^{\frac{1}{2}}}{k} \sum_{l=0}^{L}(2 l+1) \approx 2 L^{2} \sim C s^{\rho} \ln ^{2} s .
$$

Конечный результат, так называемое ограничение Фруассара, получается с помощью оптической теоремы:

$$
\sigma_{\text {tot }}(s) \sim \frac{\mathcal{I}(s, 0)}{s} \leqslant C s^{\rho-1} \ln ^{2} s
$$

Эта оценка сверху для нелокальной теории при $\rho=1$ совпадает с ограничением Фруассара локальной теории:

$$
\sigma_{\text {tot }}(s) \leqslant C \ln ^{2} s
$$

Благодарности. Работа была поддержана грантом РФФИ № 01-02-17200.

\section{Список литературы}

[1] P. Иден. Соударения элементарных частиц при высоких энергиях. М.: Наука, 1970.

[2] P. D. B. Collins and E. J. Squires. Regge Poles in Particle Physics. Berlin: Springer, 1968.

[3] В.Я. Файнберг, М.З. Иофа. ЖЭТФ. 1969. Т. 56. С. 1644; ТМФ. 1969. Т. 1. С. 187.

[4] М. А. Соловьев. Тр. ФИАН. 1993. Т. 209. С. 121.

[5] G. V. Efimov, M. A. Ivanov. Quark Confinement Model of Hadrons. Bristol: IOP, 1993.

[6] G. V. Efimov and S. N. Nedelko. Phys. Rev. D. 1995. V. 51. P. 176.

[7] G. V. Efimov and G. Ganbold. Meson Spectrum and Analytic Confinement. hep-ph/0103101.

[8] Г. В. Ефимов. Нелокальные взаимодействия квантованных полей. М.: Наука, 1977.

[9] V. Ya. Fainberg and M. Z. Iofa. Nuovo Cimento A. 1971. V. 5. P. 275. 